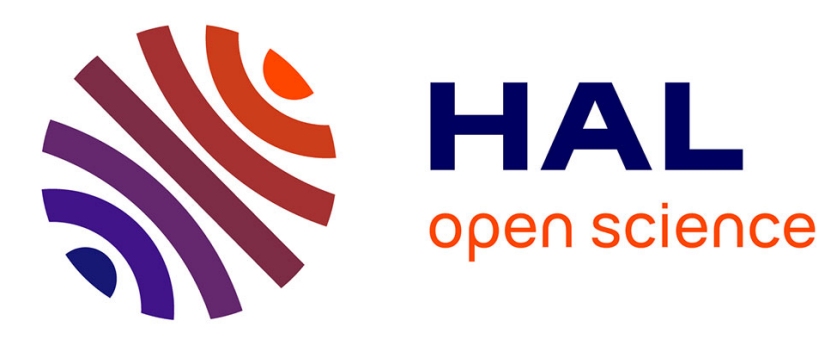

\title{
Particle rotation speeds up capillary interactions
}

\author{
J. Hemauer, M. Qiu, J. J Feng, J.-C. Loudet
}

\section{To cite this version:}

J. Hemauer, M. Qiu, J. J Feng, J.-C. Loudet. Particle rotation speeds up capillary interactions. European Physical Journal E: Soft matter and biological physics, 2021, 44 (3), 10.1140/epje/s10189021-00025-w . hal-03498678

\section{HAL Id: hal-03498678 \\ https://hal.science/hal-03498678}

Submitted on 21 Dec 2021

HAL is a multi-disciplinary open access archive for the deposit and dissemination of scientific research documents, whether they are published or not. The documents may come from teaching and research institutions in France or abroad, or from public or private research centers.
L'archive ouverte pluridisciplinaire HAL, est destinée au dépôt et à la diffusion de documents scientifiques de niveau recherche, publiés ou non, émanant des établissements d'enseignement et de recherche français ou étrangers, des laboratoires publics ou privés. 


\title{
Particle rotation speeds up capillary interactions
}

J. Hemauer ${ }^{a, b}$, M. Qiu ${ }^{b}$, J.J. Feng ${ }^{b, c}$, and J.-C. Loudet ${ }^{b, d, 1}$

${ }^{a}$ Technical University of Munich, Department of Mechanical Engineering, Garching, 85748, Germany

${ }^{b}$ University of British Columbia, Department of Mathematics, Vancouver, BC, V6T 1Z2,

Canada

${ }^{c}$ University of British Columbia, Department of Chemical and Biological Engineering, Vancouver, BC, V6T 1Z3, Canada

${ }^{d}$ University of Bordeaux, CNRS, Centre de Recherche Paul Pascal (UMR 5031), F-33600 Pessac, France

\begin{abstract}
We use dynamic numerical simulations to investigate the role of particle rotation in pairwise capillary interactions of particles trapped at a fluid interface. The fluid interface is modeled with a phase-field method which is coupled to the Navier-Stokes equations to solve for the flow dynamics. Numerical solutions are found using a finite element scheme in a bounded two-dimensional geometry. The interfacial deformations are caused by the buoyant weight of the particles, which are allowed to both translate and rotate due to the capillary and viscous forces and torques at play. The results show that the capillary attraction is faster between freely rotating particles than if particle rotation is inhibited, and the higher the viscosity mismatch, the greater the effect. To explain this result, we analyze the drag force exerted on the particles and find that the translational drag force on a rotating particle is always less than its non-rotating counterpart due to attenuated velocity gradients in the vicinity of the particle. We also find that the influence of interfacial deformations on particle rotation is minute.
\end{abstract}

Keywords: Two-phase flows, particle/fluid flow, phase-field simulations, interfacial deformations, capillary interaction, rotating particle, drag force.

\footnotetext{
${ }^{1}$ Email: jean-christophe.loudet@u-bordeaux.fr
} 


\section{Introduction}

Capillary interactions between particles trapped at fluid interfaces have been extensively investigated over many decades. Besides fundamental interest, they are known to serve as a very promising self-assembly route for a great variety of objects attached to fluid interfaces, ranging in size from tens of nanometers up to a few millimeters. Such systems are currently being exploited to design materials via diverse colloid-related technologies [1].

As is well-established, capillary interactions originate from the overlap of interfacial deformations that might occur in the vicinity of the floating objects [2]. These interfacial deformations, which cost interfacial energy, may arise from gravity, electrostatics, particle shape anisotropy or from physical and/or chemical inhomogeneities on the particle surface. Capillary interactions can be anisotropic, attractive or repulsive, and feature interaction energies much greater than the thermal energy, $k_{B} T[2,3,4,5,6,7]$. A myriad of colloidal structures, either ordered or disordered, densely-packed or loosely connected, can emerge from these interactions $[5,6,7,8]$. Such structures can also be dynamically reconfigured by applying external fields [9].

While the static properties of capillary interactions are relatively well-understood, hydrodynamic interactions of interfacial particles have been less investigated. For instance, the presence of flow around the moving particles generates viscous drag that, together with the capillary forces, controls the kinetics of the self-assembled structures.

Knowledge of the hydrodynamic properties involving two or more interfacial particles is still far from complete today. A handful of studies, using both experiments and theory, have attempted to characterize the hydrodynamic interaction for a pair of interfacial particles approaching each other $[10,11,12,13]$. In this case, the hydrodynamic resistance is a function of the interparticle distance, $r$, because of variable fluid drainage between the moving particles. And the drag dependence on $r$ interferes with that of the capillary interaction force. Despite using approximate bulk formulas for the fluid drainage, the previous studies have achieved a relatively good agreement between experiments and calculations. 
The above studies have focused on the translational motion of the interacting particles. Yet, interfacial particles can rotate as well around an axis perpendicular to their translating direction because of nonzero torques originating from, e.g., a viscosity mismatch or interfacial deformations. Furthermore, particle rotation is expected to have an influence on hydrodynamic interactions since it modifies the flow structure around the moving particles.

The first three-dimensional dynamic simulations of capillary interactions by Singh \& Joseph [14] do not mention any particle rotation in the results, and this subject was only recently brought up by Dörr \& Hardt [15]. These authors used a perturbation approach to analyze the behavior of spherical particles translating along an interface between fluids of very different viscosities under the action of an external force. A pinned contact line was assumed on the particle surface, as it often occurs in experiments $[16,17]$. In this case, a nonzero viscous torque makes the spheres rotate until the hydrodynamic torque is balanced by a capillary torque arising from interfacial deformations. As a result, the driven spheres are predicted to behave as capillary dipoles.

However, the situation where both interfacial particles rotate and translate whilst interacting with unpinned contact lines has never been addressed so far. Das et al. [18] recently considered an unpinned contact line and a fluid slip model to simulate both the translational and rotational dynamics of a sphere straddling an air-liquid interface in a thin film geometry. But the study was restricted to a single particle without any interfacial deformation. What role does particle rotation play in pairwise capillary interactions? How does it alter drag forces, and how is it influenced by interfacial deformations? These are the open questions that we attempt to address in the present study.

In this work, we focus on capillary interactions between spinning and translating particles using numerical simulations in two dimensions (2D). A 2D approach is justified here to explore the main qualitative trends. We employ a diffuse interface method, based on a phase-field model, to describe the interfacial dynamics. One of the prime advantages of such models over other approaches is their natural ability to handle contact line motion thanks to intrinsic diffusive processes $[19,20,21,22,23]$. When the phase-field model is 
combined with classical conservation equations for fluid mass and momentum, and complemented by particles' equations of motion, a full description of the system dynamics can be achieved. By comparing the behaviors of freely rotating and non-rotating particles, we find that particle rotation speeds up capillary interactions because of reduced translational drag.

\section{Geometries and parameters}

In this section, we specify the parameters and the two geometries employed in our simulations (Fig. 1). The first one, referred to as problem A hereafter, deals with the capillary pair interaction configuration, whereas the second one, referred to as problem B, is dedicated to the computation of drag forces exerted on a single spinning and translating particle straddling the interface. As we will see below, problem B is essential to understanding the results obtained in problem A since the interacting particles necessarily experience drag forces when moving at the interface. In both problems, we compare the behavior of rotating cylinders to that of non-rotating ones to single out the effect of rotation.

In problem A (Fig. 1a), two identical, infinitely long circular cylinders of radius $R$ and density $\rho_{p}$ are trapped at the interface between two Newtonian fluids of different densities $\left(\rho_{1}, \rho_{2}\right.$ with $\left.\rho_{2}>\rho_{1}\right)$ and dynamic viscosities $\left(\mu_{1}, \mu_{2}\right)$. The whole system is confined in a box of length $L$ and height $H$. The fluid interface is deformed around the cylinders, because of their weight, and the overlap of the resulting interfacial deformations drives an attractive capillary interaction until the particles contact. The characteristic length over which the deformation generated by a particle decays is given by the capillary length $L_{c}=\sqrt{\sigma / g \Delta \rho}$, where $\sigma$ is the constant interfacial tension between the two fluids, $g$ is the gravitational acceleration, and $\Delta \rho=\rho_{2}-\rho_{1}$. The competition between gravity and capillarity is more commonly discussed in terms of the dimensionless Bond number $B o=\left(R / L_{c}\right)^{2}$. In our work, $B o<1$, meaning that capillary forces will play an important role and the fluid interface is not strongly deformed. The cylinders are free to move in both the $x$ - and $y$-directions and can rotate as well with respect to their center of mass. 
The fluid interface is kept horizontal at the left and right bounding walls of the domains. The contact angle at the three-phase contact lines (cf. Fig. 1) is equal to the equilibrium contact angle $\theta$. The simulations are performed only on half of the domain shown in Fig. 1a because of symmetry.

Problem B concerns drag force calculations (Fig. 1b). We use the same setup as in our previous work [24], where only one cylinder (or disc) is trapped at the fluid interface. The main difference is that the cylinder will now be allowed to rotate freely, as in problem A. Instead of moving the cylinder horizontally across the interface, we use a reference frame attached to the cylinder so that far from it, both liquids flow with a constant velocity $U_{0}$ in the direction of the $x$-axis. In this case, the upper and lower bounding plane walls should also move with the same velocity $U_{0}$ from left to right in their own planes. In contrast to problem A, the cylinder's center of mass is kept midway across the fluid interface. In most cases, the fluid interface will be deformed around the cylinder, featuring either an upward or a downward meniscus depending on the prescribed contact angle value. The fluid interface is kept horizontal at the inlet and outlet in all cases.

For both problems $\mathrm{A}$ and $\mathrm{B}$, we use the cylinder radius $(R)$, the capillary velocity $\left(U_{\text {cap }}=\sigma / \mu_{2}\right)$, and the capillary time $\left(t_{\text {cap }}=R / U_{\text {cap }}\right)$ as the characteristic length, velocity and time scales, respectively, if not otherwise stated. Viscosity and density ratios are defined as $\mu^{*}=\mu_{1} / \mu_{2}, \rho^{*}=\rho_{1} / \rho_{2}, \rho_{p}^{*}=\rho_{p} / \rho_{2}$, respectively, where $\rho_{p}$ is the particle density. With the above scaling, table 1 lists all the physical and geometrical parameters used in both problems. The dimensional baseline values are specified in the table caption.

\section{Governing equations and numerical method}

\subsection{Two-phase flow modeling}

For both problems A and B, we opt for a diffuse interface method, based on the concept of "phase-field" (PF), to account for the fluid interface motion, combined with the NavierStokes (NS) equations to describe the flow. As pointed out in the introduction, the choice of the PF model was primarily motivated by its intrinsic ability to simulate contact line 
(A)
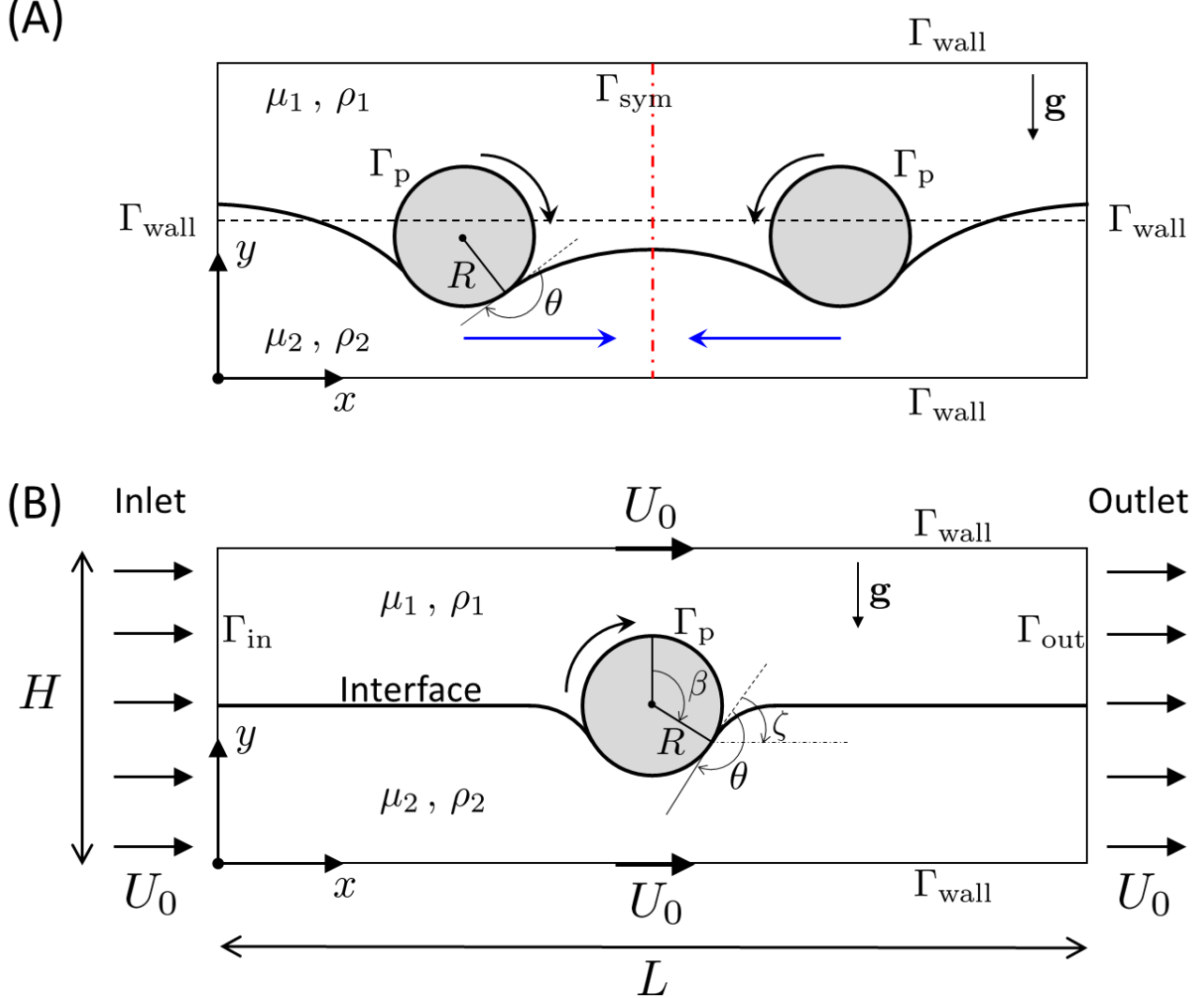

Figure 1: Two-dimensional geometries of the two problems investigated in this work. (A) Geometry for the computation of the pair capillary interaction between floating cylinders at a fluid interface (problem A). The vertical red dashed-dot line marks the symmetry plane $\left(\Gamma_{\text {sym }}\right)$ and the solid blue arrows represent the capillary attraction between the two particles due to interfacial deformations. The curved arrows indicate particle rotation. Symbols: $\theta$ : contact angle, g: gravitational acceleration. (B) Geometry for the computation of two-phase flow drag on a circular rotating cylinder confined at a fluid interface between two plane walls (problem B). Symbols: $\beta$ : floating angle, $\zeta$ : interface slope at the contact line. See table 1 for other symbol definitions. 
Table 1: Definitions and dimensionless values (denoted with $*$ ) of the parameters used in problems $A$ and $B$. In the following, the letters $A$ and $B$ in the superscript refer to problems $A$ and $\mathrm{B}$, respectively. $U_{x}^{\max }$ is the maximum particle translational velocity. Dimensional baseline values: $R=1 \mathrm{~mm}, \sigma=0.01 \mathrm{~N} / \mathrm{m}, \mu_{2}=0.1$ Pa.s, $\rho_{2}=10^{3} \mathrm{~kg} \cdot \mathrm{m}^{-3}$.

\begin{tabular}{l|cc}
\hline \hline Parameter & Symbol & Value \\
\hline \hline Cylinder radius & $R^{*}$ & 1 \\
Box length & $\mathrm{L} / \mathrm{R}$ & $32^{A}, 30^{B}$ \\
Box height & $\mathrm{H} / \mathrm{R}$ & $16^{A}, 20^{B}$ \\
Inlet/outlet velocity & $U_{0}^{*}$ & $2 \times 10^{-4}$ \\
Viscosity ratio & $\mu^{*}=\mu_{1} / \mu_{2}$ & {$[0.05-20]^{A},[0.05-1]^{B}$} \\
Fluid density ratio & $\rho^{*}=\rho_{1} / \rho_{2}$ & 0.9 \\
Particle density ratio & $\rho_{p}^{*}=\rho_{p} / \rho_{2}$ & 1.05 \\
Interfacial tension & $\sigma^{*}$ & 1 \\
Contact angle & $\theta$ & $100^{\circ} A\left[45^{\circ}-135^{\circ}\right]^{B}$ \\
Reynolds number & $R e=\rho_{2} U_{x}^{\max } R / \mu_{2}$ & $\sim 10^{-3}$ \\
Capillary number & $C a=\mu_{2} U_{x, \max } / \sigma$ & $\lesssim 10^{-3}$ \\
\hline \hline
\end{tabular}


motion without any stress singularity or ad hoc fluid slip models [19, 20, 21, 22, 23]. The details of the coupled phase-field/Navier-Stokes equations have already been described in our previous work [24] and by a number of authors [23, 25, 26, 27]. We refer the readers to these works for all the technicalities. In the following, we only give a brief account of some key features of the model.

In the PF formulation, the two fluid components, which are otherwise considered immiscible, are allowed to mix within a thin interfacial region. A PF variable, $\phi$, is introduced to characterize the two different phases. The key idea is that $\phi$ changes rapidly but continuously within the thin interfacial region, whose thickness is controlled by the capillary width $\epsilon$. In the two fluid bulks, $\phi= \pm 1$, and the fluid-fluid interface may be defined by the contour level $\phi=0$. The interfacial dynamics is modeled by an evolution equation for $\phi$, the Cahn-Hilliard $(\mathrm{CH})$ equation $[25,26]$

$$
\frac{\partial \phi}{\partial t}+\mathbf{v} \cdot \nabla \phi=\nabla \cdot(\gamma \nabla G)
$$

where $G=\lambda\left[-\nabla^{2} \phi+\left(\phi^{2}-1\right) / \epsilon^{2}\right]$ is the chemical potential, $\lambda$ the mixing energy density, $\gamma$ a diffusion parameter called the mobility (assumed to be constant), and $\mathbf{v}$ the fluid velocity. The fact that the interface can move by diffusive processes is essential to handle contact line dynamics, as aforesaid. It is also worth stressing that the resolution of the interface thickness is of paramount importance in the PF method (see below) [23, 26, 28].

Assuming incompressible fluids, the pressure $(p)$ and velocity $(\mathbf{v})$ fields in both phases are described by the standard Navier-Stokes equations

$$
\begin{aligned}
\boldsymbol{\nabla} \cdot \mathbf{v} & =0 \\
\rho(\phi)\left(\frac{\partial \mathbf{v}}{\partial t}+\mathbf{v} \cdot \boldsymbol{\nabla} \mathbf{v}\right) & =-\boldsymbol{\nabla} p+\boldsymbol{\nabla} \cdot \underline{\boldsymbol{\sigma}}^{\boldsymbol{v}}+\rho(\phi) \mathbf{g}+G \boldsymbol{\nabla} \phi
\end{aligned}
$$

where the last body force term in Eq. (3) $(G \boldsymbol{\nabla} \phi)$ is the diffuse-interface equivalent of the interfacial tension $[25,28] . \underline{\boldsymbol{\sigma}}^{\boldsymbol{v}}=\mu(\phi)\left[\boldsymbol{\nabla} \mathbf{v}+(\boldsymbol{\nabla} \mathbf{v})^{T}\right]$ is the viscous stress tensor, $\mathbf{g}$ is the gravitational acceleration, and $\rho(\phi)$ (resp., $\mu(\phi))$ is the density (resp., the viscosity) of the two-phase system given by: $\rho(\phi)=\frac{1+\phi}{2} \rho_{1}+\frac{1-\phi}{2} \rho_{2}$, and $\mu(\phi)=\frac{1+\phi}{2} \mu_{1}+\frac{1-\phi}{2} \mu_{2}$.

Classical no-slip boundary conditions for $\mathbf{v}$ are imposed on all bounding walls and cylinders, together with a no penetration and vanishing shear stress condition on $\Gamma_{\text {sym }}$ 
(Fig. 1a). More precisely, for rotating cylinders, we impose $\mathbf{v}=\mathbf{U}+R \omega \hat{\mathbf{t}}$ on $\Gamma_{p}$, where $\mathbf{U}$ (resp., $\omega$ ) is the cylinder's translational (resp., angular) velocity, and $\hat{\mathbf{t}}$ is the tangential unit vector along the particle contour. For non-rotating cylinders, the condition $\mathbf{v}=\mathbf{U}$ is prescribed on $\Gamma_{p}$. Within the $\mathrm{PF}$ model, we enforce the equilibrium value of the contact angle, $\theta$, at the three-phase contact lines of cylinders via the geometric boundary condition, $\boldsymbol{\nabla} \phi \cdot \hat{\mathbf{n}}=|\boldsymbol{\nabla} \phi| \cos \theta$, where $\hat{\mathbf{n}}$ is the outward unit normal vector to $\Gamma_{p}$. We also impose a $90^{\circ}$-contact angle on $\Gamma_{\text {wall }}$ (left side) and $\Gamma_{\text {sym }}$ (Fig. 1a), and on $\Gamma_{\text {in, out }}$ (Fig. 1b), meaning that the interface is flat there but it is free to move up or down.

\subsection{Particles' equations of motion}

In problem A (Fig. 1a), the two cylinders are free to translate along the $x$ and $y$ directions and rotate around an axis perpendicular to the $x y$-plane. Their translational $\left(\mathbf{U}=\left(U_{x}, U_{y}\right)\right)$ and angular $(\omega)$ velocities are governed by Newton's equations of motion involving viscous and capillary forces and torques in the presence of gravity (see Sec. 2 of Supplementary Information (SI) for details). The viscous and capillary forces are computed by integrating the corresponding viscous and capillary tractions, respectively, along the 2D particle contour. Similarly, the viscous torque is obtained by a contour integration of the viscous stress moment. Note that for a spherical particle (or a cylinder/disc in 2D), provided that the interfacial tension and the contact angle are constant, the capillary torque always vanishes regardless of the position and shape of the contact line. This has been confirmed in both literature [29] and our calculations. Therefore, only the viscous torque needs to be considered here. Finally, two kinematic equations link the cylinders' positions (resp., angular displacements) to their velocities (resp., angular velocities): $d \mathbf{r}_{p} / d t=\mathbf{U}$ and $d \alpha / d t=\omega$, where $\mathbf{r}_{p}=\left(x_{p}, y_{p}\right)$ denotes the location of one cylinder's center of mass and $\alpha$ is its angular displacement. Note that the above description is valid for both translating and rotating cylinders. For the non-rotating ones, of course, we only solve the equations of motion for the translation.

In problem B, the cylinder's center of mass is fixed and the particle may be allowed to freely rotate with an angular velocity, which is determined by the viscous torque exerted 
on it due to the incoming flow (Fig. 1b). In this case, only the particle torque equation is solved, and the cylinder contour rotates with a velocity equal to $R \omega \hat{\mathbf{t}}$.

\subsection{Numerical method}

The fluid equations, and their associated boundary conditions, together with the particles' equations of motion, were solved numerically with the finite element computational software COMSOL Multiphysics ${ }^{\circledR}$ [30]. We used the coupled laminar two-phase flow/phasefield (Cahn-Hilliard) modules combined with an ordinary differential equations module to compute all the unknowns, i.e. $\mathbf{v}, p, \phi, \mathbf{U}, \mathbf{r}_{p}, \omega, \alpha$, at each time step. Furthermore, for problem A, we employed the built-in moving mesh module of COMSOL based on an Arbitrary Lagrangian-Eulerian (ALE) scheme to follow and resolve the particles' motion as they attract one another through capillary interaction. Since the particles travel over long distances, typically $\sim 5-10 R$, mesh deformation is substantial (fig. S1a in SI). In order to maintain a high-quality mesh and avoid inverted elements, we used the automatic remeshing feature of COMSOL (fig. S1b in SI). We designed a nonuniform triangular mesh fitted with subdomains to appropriately resolve the fluid interface as it moved within the domain. The subdomains were uniformly meshed with a mesh size adjusted so that the interfacial thickness, which is on the order of $4 \epsilon$, contained at least 8 elements. According to the criterion defined in [28], the latter condition ensures a sufficient resolution of the fluid interface, which is a crucial issue for the PF method [22, 23, 26, 28]. Outside of the subdomains, the mesh size was much coarser since the fluid interface never wandered into these areas (fig. S1b in SI). Finally, the value of the mobility parameter $(\gamma)$ was adjusted according to the guidelines reported in [22] to ensure convergent results with moving contact lines. Additional numerical details can be found in Sec. 1 of SI.

\section{Results and discussion}

We now address the core issue of the present work, i.e. the influence of particle rotation on pairwise capillary interactions. We will first present the central result of the paper, 
for problem A, together with an examination of the role of the viscosity ratio. Then we study the drag force on a rotating interfacial cylinder (problem B), to seek an explanation for the findings in problem A.

\subsection{Capillary interaction with rotating particles}

In problem A (Fig. 1a), initially, the centers of mass of the cylinders lie in the plane of the fluid interface and their center-to-center distance is $d=L / 2-2 \Delta_{x}$, where $\Delta_{x}$ is a small lateral shift ensuring that each cylinder is closer to the symmetry plane $\left(\Gamma_{\mathrm{sym}}\right)$ than to the side wall. Indeed, if $d=L / 2$, the menisci on both sides of a cylinder will be symmetric and no capillary interaction would occur initially. If $d>L / 2$, each cylinder would be attracted towards its respective side wall, and the two would move apart from each other.

Before releasing the cylinders, the fluid interface is flat everywhere and makes a $90^{\circ}$ contact angle at the contact lines. From the values listed in table 1 , we get $L_{c}^{*} \simeq 3.19$ and $B o=0.098$. The contact angle is kept constant and equals $100^{\circ}$ throughout this section. Furthermore, we have chosen a box size of $(H, L)=(16 R, 32 R)$, which makes sure that, (i) the upper and lower confining walls are several particle radii away from the cylinder, so we are not dealing with a highly confined geometry, and (ii) the lateral extension of the fluid interface covers a few times the capillary length. We have checked that the use of larger boxes (e.g. $(H, L)=(20 R, 40 R))$, besides being more computationally expensive, only shifts the capillary interaction process to longer times, without altering the result qualitatively (the magnitude of the capillary force near contact differs by less than $4 \%$ between box sizes $(H, L)=(16 R, 32 R)$ and $(H, L)=(20 R, 40 R))$. Note that, as in Loudet et al. [24], we are not interested in eliminating the influence of confining walls, which is nearly impossible in 2D low-Reynolds number flows [31]. Also, for most cases, we have chosen either $C n=0.04$ or $C n=0.02$, which turned out to be good values realizing a trade-off between precision and reasonable computing time. In what follows, we present numerical data obtained with a moderately small viscosity ratio $\mu^{*}=0.1$, essentially for illustrative purposes. But other values of $\mu^{*}$ will be tested in the next section.

Upon releasing the cylinders, they first tend to sink into the lower phase since they are 
denser than the surrounding fluids. After a transient period, the vertical force balance is achieved and the particles' buoyant weight is balanced by the vertical component of the surface tension force acting at the contact line. The fluid interface is distorted and satisfies the contact angle condition. Because of interfacial distortions, the particles start moving towards each other in the $x$-direction through capillary interaction. This motion stirs flow in the vicinity of the particles, which now experience a viscous drag force opposing their displacement. Moreover, because of interfacial deformations, which yield an up-down asymmetry in the flow pattern [24], the drag force acting on the particles yields a nonzero viscous torque as well. The reason for this is that the drag force's line of action does not generally run through the particle's center of mass, even if $\mu^{*}=1$. Consequently, the particle rotates. Of course, a viscosity mismatch between the two fluids $\left(\mu^{*} \neq 1\right)$ will further alter the viscous torque and hence the particle rotation, as pointed out by Dörr \& Hardt [15].

Our numerical results are summarized in Fig. 2. In Fig. 2a, we have plotted the $x$ component of the particle velocity $\left(U_{x}^{*}\right)$ as a function of time $\left(t^{*}\right)$ for both a rotating and a non-rotating cylinder. All our results are presented for the left cylinder in Fig. 1a.

The key result of Fig. 2a is the effect of particle rotation on the speed of the capillary interaction. With the used parameters, the interaction time is reduced by about $10 \%$ with rotating particles, with a roughly $20 \%$-increase in the particle translational velocity near contact. $U_{x}^{*}$ strongly accelerates prior to dropping abruptly just before the particles collide, probably because of strong lubrication forces due to the squeezed thin film inbetween the particles.

The corresponding dimensionless angular velocity $\left(\omega^{*}\right)$ and angular displacement $(\alpha)$ versus time are plotted in Fig. 2b. We used $U_{x}^{\max } / R$ as the characteristic angular velocity so that $\omega^{*}=\omega /\left(U_{x}^{\max } / R\right)$. Fig. $2 \mathrm{~b}$ shows that $\omega^{*}=O(1)$, especially close to contact, meaning that we are in a regime where the particle rotation is significant and is part of the physical description of the system. With our sign convention, a negative (resp., positive) value of $\omega^{*}$, or $\alpha$, implies a clockwise (resp., counter-clockwise) rotation of the left cylinder. Since it moves to the right, fluid is drained out in the opposite direction (Fig. 3). In this 

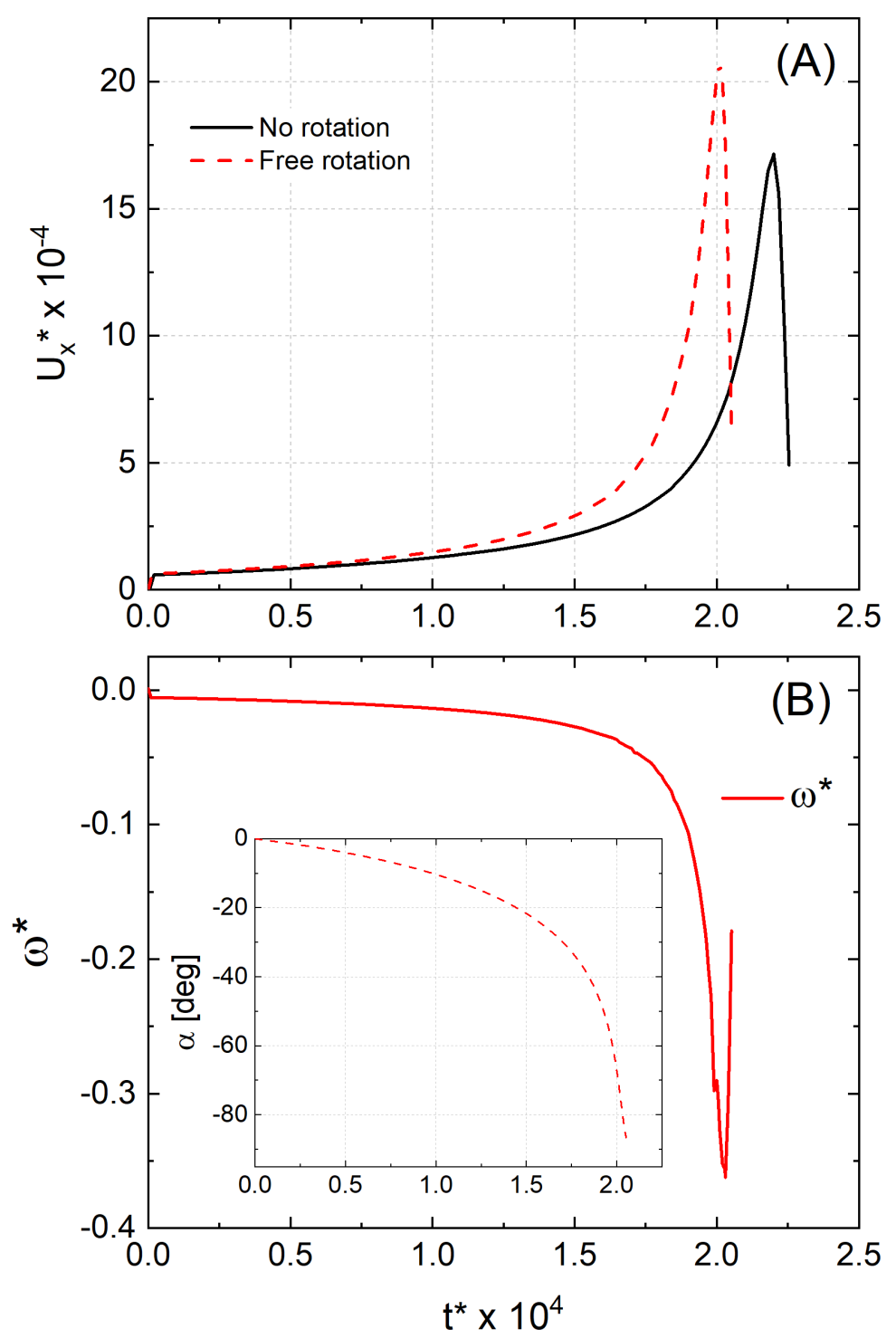

Figure 2: Results of dynamic simulations of the capillary pair interaction with and without particle rotation. (A) Particle velocity $\left(U_{x}^{*}=U_{x} /\left(\sigma / \mu_{2}\right), x\right.$-component $)$ as a function of time $\left(t^{*}=t /\left(\mu_{2} R / \sigma\right)\right)$. The particles' rotation speeds up the interaction kinetics. (B) Angular velocity $\left(\omega^{*}=\omega /\left(U_{x}^{\max } / R\right)\right)$ as a function of time $t^{*}$. The inserted graph represents the time evolution of the corresponding angular displacement $(\alpha)$. Parameters: $\mu^{*}=0.1, \theta=100^{\circ}$, $C n=0.04$, see also table 1 . 


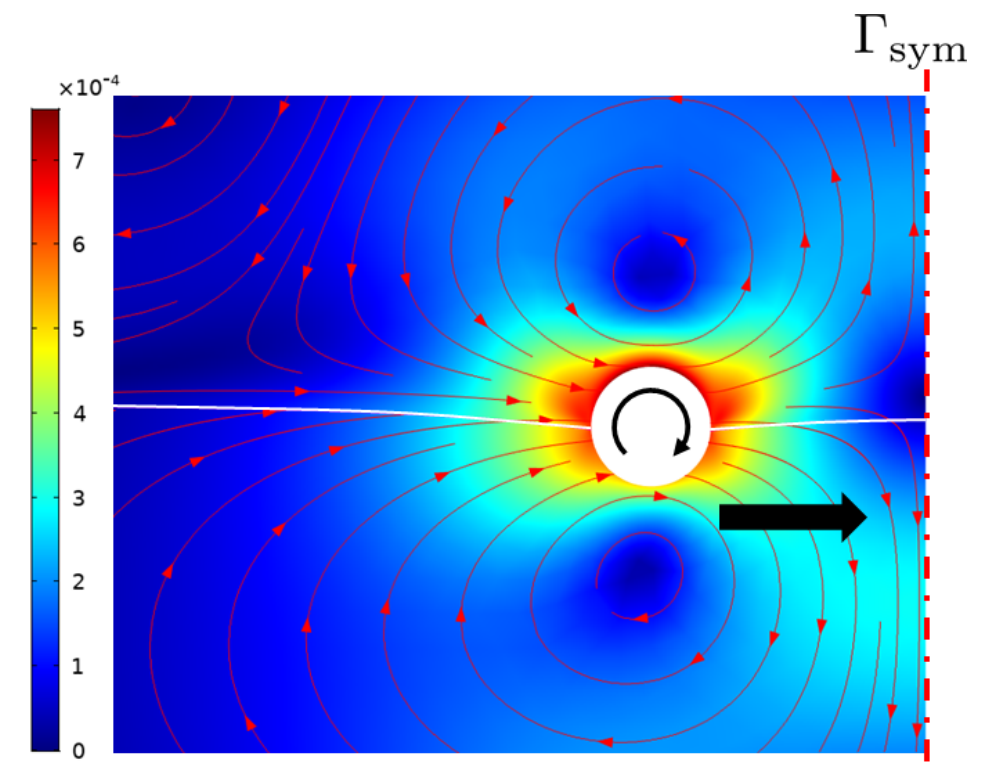

Figure 3: Snapshot of the velocity field for rotating particles undergoing a capillary attraction (time $t^{*}=5.6 \times 10^{4}$; final time at contact: $t^{*}=5.85 \times 10^{4}$ ). Only the left half of the computational domain is considered due to the symmetry axis $\Gamma_{\text {sym }}$ (see Fig. 1a). The particle translates to the right whilst rotating clockwise (curved black arrow). The streamlines are represented by red arrow lines whereas the solid white line indicates the fluid interface $(\phi=$ $0)$. The color bar gives the scaled velocity amplitude $\left(|\mathbf{v}| / U_{\text {cap }}\right.$, see Sec. 2). Parameters: $\mu^{*}=0.05, \theta=100^{\circ}, C n=0.04$, see also table 1 .

case, the clockwise rotation of the left cylinder during the interaction process arises from greater viscous stresses exerted on its bottom submerged part because of a higher fluid viscosity there $\left(\mu^{*}=0.1\right)$. Swapping the fluid viscosities, i.e. having $\mu^{*}>1$, would result in a counter-clockwise rotation of the left cylinder for the same reasons (see Sec. 4.2). Recall that the capillary torque is zero in all our simulations (Sec. 3.2), and that the particle rotation is only driven by viscous forces.

The dimensionless capillary interaction force $F_{c}^{*}=F_{c} /\left(\sigma B o^{2}\right)$, where $F_{c}$ is obtained by integrating the capillary traction over the particles' contours (see Eqs. (4)-(5) in Sec. 2 of SI), is graphed in Fig. 4 as a function of time $\left(t^{*}\right)$ for both rotating and non-rotating cylinders. Since the particles' rotation speeds up their attraction, the profiles are shifted in time but the magnitude of $F_{c}^{*}$ remains the same in both cases near contact. Actually, 


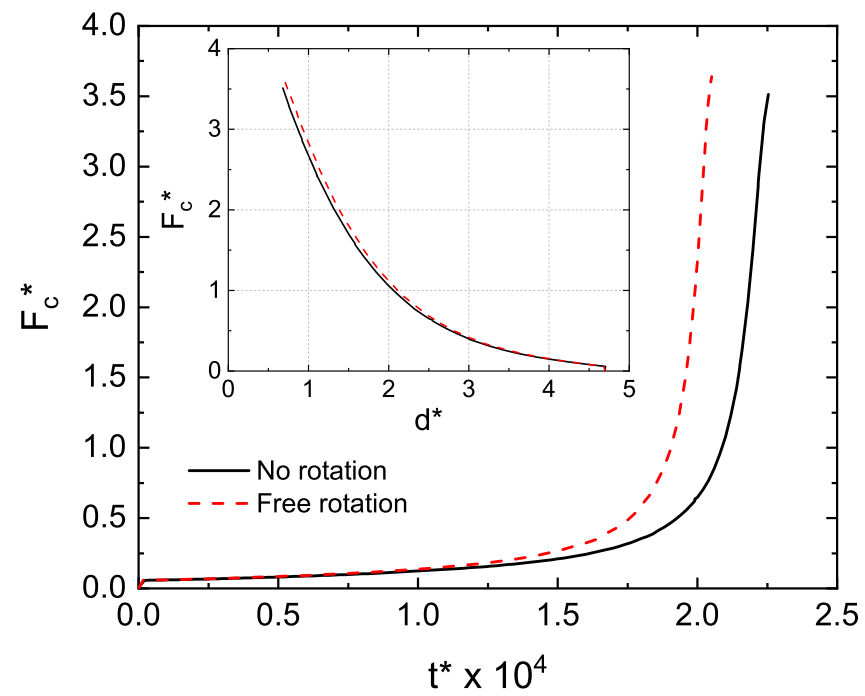

Figure 4: Capillary interaction force $\left(F_{c}^{*}=F_{c} /\left(\sigma B o^{2}\right)\right)$ for rotating and non-rotating cylinders as a function of time $\left(t^{*}\right)$. Inset: $F_{c}^{*}$ versus the center-to-center distance $\left(d^{*}=d / L_{c}\right)$. Parameters: $\mu^{*}=0.1, \theta=100^{\circ}, C n=0.04$, see also table 1 .

upon plotting $F_{c}^{*}$ versus $d^{*}=d / L_{c}$, we see that the magnitude of the interaction force is hardly altered by the particles' rotation all along the interaction trajectory (see inset in Fig. 4). This result further suggests that the interfacial profiles for rotating and nonrotating cylinders should be essentially identical within our operating conditions. Indeed, Fig. S2 (SI) confirms that the profiles are almost indistinguishable in both situations.

The fact that $F_{c}^{*}$ is insensitive to particle rotation implies that the drag force exerted on the particle must be reduced by the rotation to yield a faster interaction (Fig. 4). This conjecture calls for in-depth drag force calculations with both rotating and non-rotating particles, which will be described in problem B below (Sec. 4.3). But prior to changing the geometry, we first explore the influence of the viscosity ratio on the interaction dynamics.

\subsection{Influence of the viscosity ratio}

We performed additional simulations with other values of the viscosity ratio $\mu^{*}$ to examine its influence on the interaction speed. Fig. 5 summarizes the data obtained for the trans- 
lational (Fig. 5a) and angular (Fig. 5b) velocities with $\mu^{*}$ in the range [0.05-0.75]. We see that the greater the viscosity mismatch, the higher $U_{x}^{*}$ and $\omega^{*}$, and the faster the dynamics. As expected, the viscous torque exerted on the cylinders is enhanced at small $\mu^{*}$ and make them rotate faster. Consequently, the maximum angular displacement, marked as a number close to each curve in Fig. 5b, increases as $\mu^{*}$ decreases, despite a shorter interaction time. In addition, the boost in the speed of capillary attraction by particle rotation is greater for a stronger viscosity mismatch (Fig. 5a). While only a very modest difference in the interaction time exists for $\mu^{*}=0.75(<1 \%$, compare the solid black line and the red dashed one), the difference jumps to about $12 \%$ for $\mu^{*}=0.05$ (compare the solid blue curve with the green dashed one) within our operating conditions. Hence, the strongest effects would be expected for, e.g., liquid-gas interfaces.

Next, we also briefly consider the case $\mu^{*}>1$ for which the upper fluid is more viscous than the lower one, whilst keeping all the other parameters unchanged. Because of the inversion of the viscosity mismatch, Fig. S3 (SI) shows that the left cylinder now spins counter-clockwise $(\omega>0)$, as explained previously (Sec. 4.1). However, it is worth pointing out that the spinning direction does not alter the phenomenon we described above. Whether the cylinders spin clockwise or counter-clockwise, the interaction dynamics is always accelerated when compared to the non-rotating situation (see Fig. S3a in SI). In addition, swapping the viscosities makes the interaction faster, but this phenomenon is peculiar to our choice of parameter values, especially the contact angle and the buoyant weight. This is explained in detail in SI.

\subsection{Drag force with rotating particles}

In this section, we switch to problem B (Fig. 1b) and investigate the influence of particle rotation on the viscous drag force in the presence of interfacial deformations. The following simulations are motivated by the data gathered in problem A, which strongly suggest that the boost in capillary attraction by particle rotation, regardless of its sense, occurs via a reduction in the drag force.

In Fig. 1b, the cylinder is held fixed in the middle of the box as flow passes by it. The 

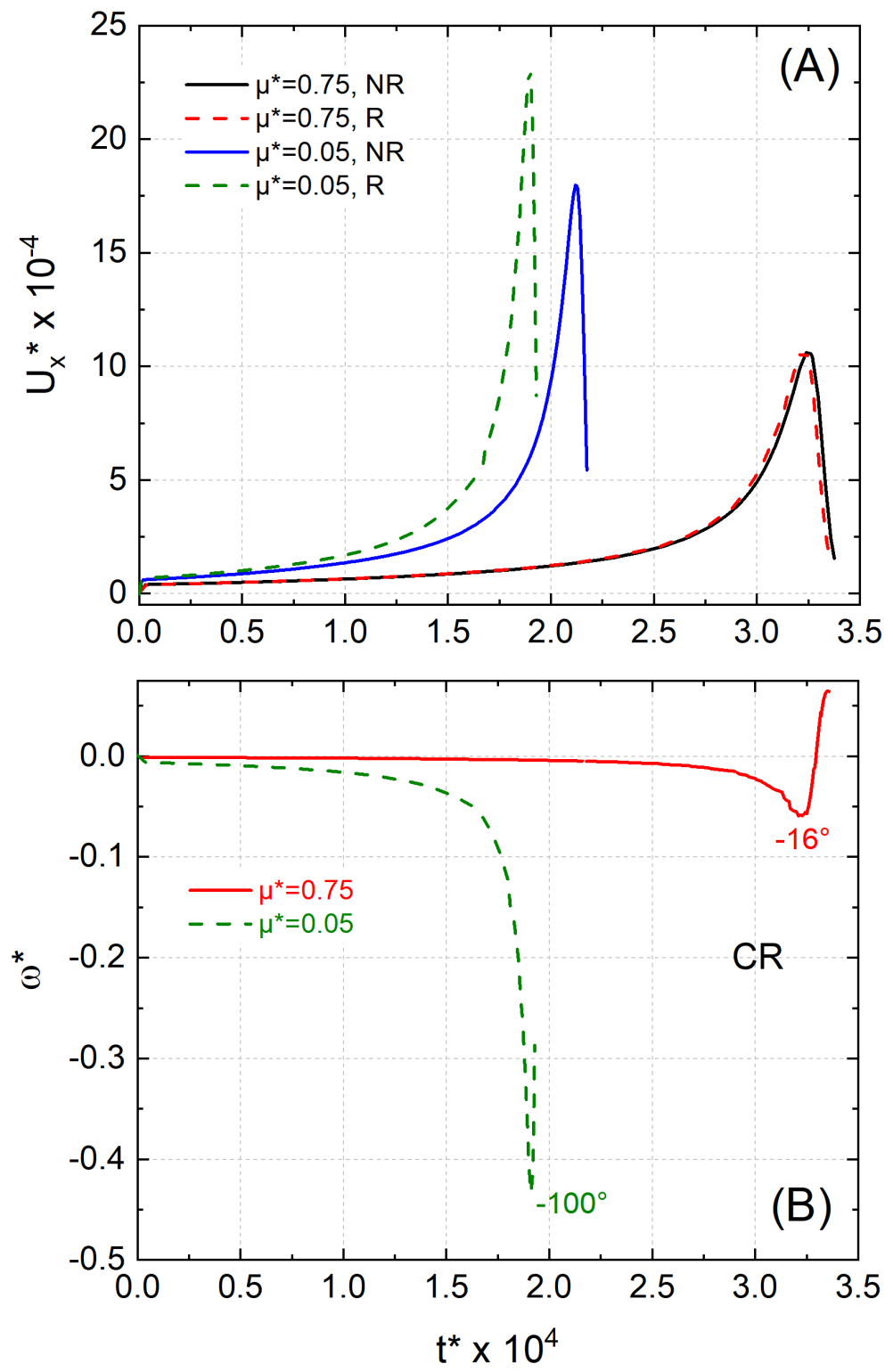

Figure 5: Influence of the viscosity ratio on the computed translational and rotational velocities of particles undergoing an attractive capillary interaction. (A) Translational velocity $\left(U_{x}^{*}\right)$ and (B) Angular velocity $\left(\omega^{*}\right)$ as a function of time $\left(t^{*}\right)$ for two viscosity ratios. In (B), the numbers next to each curve indicate the maximum angular displacement $(\alpha)$ reached at contact. NR: non-rotating; R: rotating; CR: clockwise rotation. Parameters: $\theta=100^{\circ}, C n=0.04$, see also table 1. 
contact angle is fixed to $\theta=100^{\circ}$ with $\mu^{*}$ in the range [0.05-0.75], as in problem A. As a consequence, the fluid interface is slightly deformed and exhibits a downward meniscus close to the particle. As in problem $\mathrm{A}$, we have $L_{c}^{*} \simeq 3.2$ and $B o \simeq 0.1$. The box size was fixed to $(H, L)=(20 R, 30 R)$ (table 1$)$ with $C n=0.02$ for a better accuracy but still a manageable computing time (Sec. 1 of SI).

Table 2 compares the values of the viscous drag force (Eq. (4), Sec. 2 of SI) on a rotating $\left(F_{v, r}\right)$ and a non-rotating $\left(F_{v, n r}\right)$ cylinder in the above conditions. The force values have been normalized by $\sigma B o^{2}$. While the difference between $F_{v, r}$ and $F_{v, n r}$ is not significant for $\mu^{*}=0.75, F_{v, r}$ becomes clearly less than $F_{v, n r}$ as $\mu^{*}$ decreases further and the particle rotates faster (last column in table 2 ). For $\mu^{*}=0.05$, the difference is about $8 \%$. These results confirm the conjecture put forward previously and also corroborate very well the viscosity effect illustrated in Fig. 5 for problem A (Sec. 4.2). With a non-rotating cylinder, the no-slip boundary condition requires that the fluid velocity be arrested on its surface, thereby leading to larger velocity gradients and tangential shear stresses. This effect is reduced with a rotating cylinder. The flow field around the cylinders illustrates this difference (Fig. 6). While the non-rotating cylinder features two high-shear regions above and below the cylinder (Fig. 6a), the rotating cylinder has just one such region above the cylinder (Fig. 6b). The rotation has greatly reduced the velocity gradient below it. Notably, in their recent computational work, Koplik \& Maldarelli [32] also reported that $F_{v, r}^{*}<F_{v, n r}^{*}$ for an isolated nanosphere straddling a flat liquid-vapor interface under the action of an external force.

To provide additional insight into the drag reduction for a rotating cylinder, one can compute the rate of energy dissipation, $\varepsilon$, in the entire simulation domain $(\Omega)[33]$ : $\varepsilon=\int_{\Omega} \mu_{\mathrm{av}} \Phi d \Omega$, where $\mu_{\mathrm{av}}=\frac{1+\phi}{2} \mu_{1}+\frac{1-\phi}{2} \mu_{2}$ is the average viscosity of the two-phase system in the PF model (Sec. 3.1), and $\Phi$ is the viscous dissipation function which, for an incompressible fluid and a 2D geometry, may be written as

$$
\Phi=2\left[\left(\frac{\partial v_{x}}{\partial x}\right)^{2}+\left(\frac{\partial v_{y}}{\partial y}\right)^{2}\right]+\left(\frac{\partial v_{y}}{\partial x}+\frac{\partial v_{x}}{\partial y}\right)^{2} .
$$

The values of $\varepsilon$ at steady-state, scaled by $\mu_{2} U_{0}^{2}$, are also reported in table 2 for rotating and non-rotating cylinders. Similarly to the drag force, the rate of energy dissipation is 


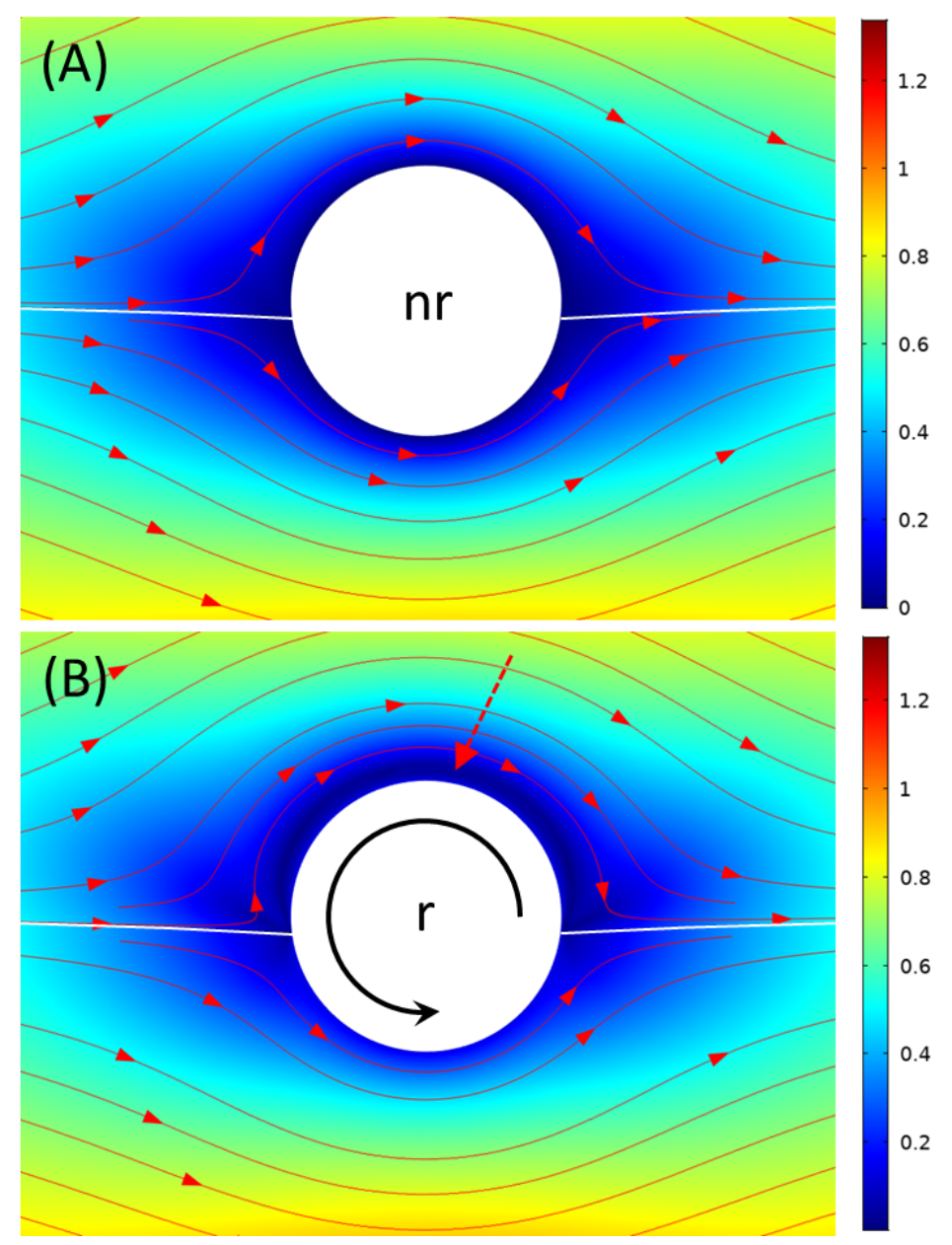

Figure 6: Steady-state streamlines in the vicinity of a non-rotating ( $\mathrm{nr}$ ) (A) and a rotating (r) (B) cylinder for problem B (Fig. 1b). The maps display the velocity magnitude scaled by the incoming flow velocity $\left(U_{0}\right)$ (cf. color scales). In $(\mathrm{A})$, the fluid velocity is zero on the particle contour, but in (B), where the cylinder rotates counter-clockwise, the area of zero-fluid velocity is only present in the upper fluid and is shifted off the particle surface (cf. the dark blue half-circle marked by the red dashed arrow). In both cases, the solid red arrows indicate the flow streamlines while the solid white line corresponds to the fluid interface $(\phi=0)$. Parameters: $\theta=100^{\circ}, \mu^{*}=0.1, C n=0.02$, see also table 1 . 
Table 2: Normalized drag force $\left(F_{v}^{*}=F_{v} /\left(\sigma B o^{2}\right)\right)$ and rate of energy dissipation $\left(\varepsilon^{*}=\right.$ $\varepsilon / \mu_{2} U_{0}^{2}$ ) for a non-rotating ( $\mathrm{nr}$ ) and rotating ( $\mathrm{r}$ ) cylinder in problem B (Fig. 1b) as a function of $\mu^{*}$. In the latter case, the values of the angular velocity $\left(\omega^{*}=\omega /\left(U_{0} / R\right)\right)$ are also reported. Both $F_{v}$ and $\varepsilon$ are smaller with a rotating cylinder than a non-rotating one. The uncertainties are $\Delta F_{v}^{*}= \pm 2.10^{-4}, \Delta \varepsilon^{*}= \pm 0.03$, and $\Delta \omega^{*}= \pm 10^{-3}$. Parameters: $\theta=100^{\circ}, C n=0.02$, see also table 1 .

\begin{tabular}{c|cc|cc|c}
\hline \hline$\mu^{*}$ & $F_{v, n r}^{*}$ & $F_{v, r}^{*}$ & $\varepsilon_{n r}^{*}$ & $\varepsilon_{r}^{*}$ & $\omega^{*}$ \\
\hline \hline 0.75 & 0.1629 & 0.1628 & 7.83 & 7.82 & 0.013 \\
0.1 & 0.1001 & 0.0945 & 4.87 & 4.50 & 0.136 \\
0.05 & 0.0952 & 0.0879 & 4.65 & 4.18 & 0.161 \\
\hline \hline
\end{tabular}

reduced in the rotating case, and the difference between $\varepsilon_{r}^{*}$ and $\varepsilon_{n r}^{*}$ grows for larger viscosity mismatches. This result further supports our interpretation above to account for the faster interaction dynamics with rotating cylinders (Sec. 4.1).

\section{Influence of interfacial deformations}

In this last section, we briefly investigate the role of interfacial deformations on particle rotation. We use the geometry of problem B as a predictive tool to see if large differences between $F_{v, r}$ and $F_{v, n r}$ can be achieved by tuning the value of the prescribed contact angle on the cylinder contour. In order to single out the effect of meniscus deformation, we only consider fluids with matched viscosities in the following, i.e. $\mu^{*}=1$. The procedure is exactly the same as the one already described in Sec. 4.3.

The results are summarized in Fig. 7 with $\theta$ in the range $45^{\circ}-135^{\circ}$. Recall that the particle's center of mass is fixed here (Fig. 1b) and, consequently, the interfacial deformations increase as $\theta$ deviates more and more from $90^{\circ}$. In Fig. 7a, the drag force for both rotating $\left(F_{v, r}^{*}\right)$ and non-rotating $\left(F_{v, n r}^{*}\right)$ cylinders are plotted as a function of the contact angle. Note that those values have been normalized with respect to the drag force 


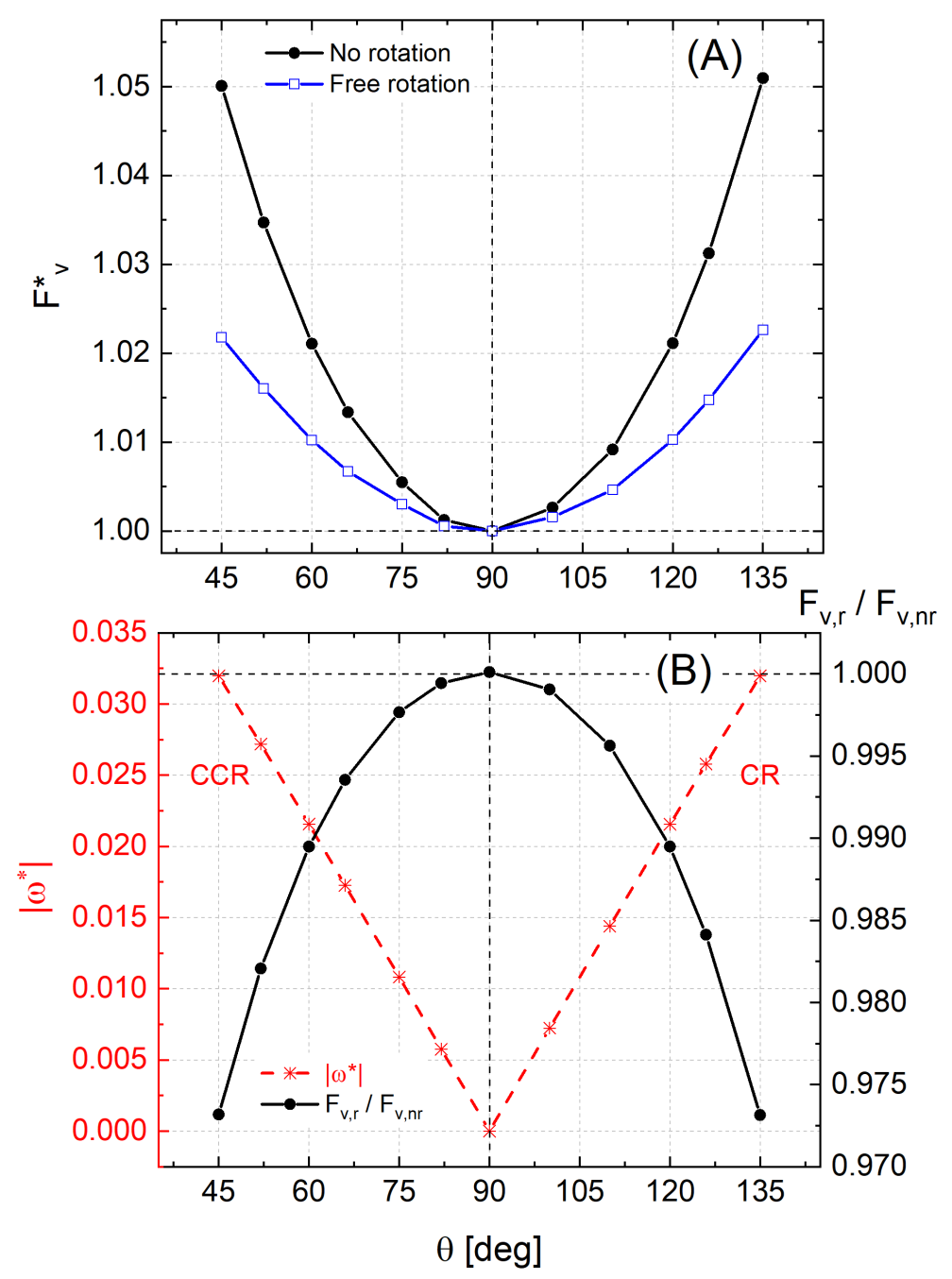

Figure 7: (A) Normalized translational drag force, $F_{v}^{*}$, as a function of the contact angle $(\theta)$ for both rotating $(r)$ and non-rotating $(n r)$ particles. The normalization was done with respect to the drag force value obtained for $\theta=90^{\circ}$ in the non-rotating case. For $\theta \neq 90^{\circ}$, we see that $F_{v, r}^{*}<F_{v, n r}^{*}$. (B) Drag force ratio, $F_{v, r} / F_{v, n r}$, and absolute value of the angular velocity $\left(\omega^{*}=\omega /\left(U_{0} / R\right)\right)$ as a function of $\theta$. It is seen that the larger $\left|\omega^{*}\right|$, the smaller the ratio $F_{v, r} / F_{v, n r}$ compared to unity. CCR: counter-clockwise rotation $\left(\theta<90^{\circ}\right)$; CR: clockwise rotation $\left(\theta>90^{\circ}\right)$. Parameters: $\mu^{*}=1, C n=0.02$, see also Fig. $1 \mathrm{~b}$ and table 1. 
calculated in the flat interface case, i.e. for $\theta=90^{\circ}$. In this way, the effect of interfacial deformations can be readily assessed by direct reading. For non-rotating cylinders, the drag force increases as soon as $\theta$ departs from $90^{\circ}$. As explained in [24], interfacial deformations yield an up-down asymmetry in the flow pattern with respect to the flat interface configuration, which results in an increased friction. And since both fluids have equal viscosities, the enhanced dissipation is symmetric with respect to $\theta=90^{\circ}$.

The corresponding data for a rotating cylinder display a similar symmetric concave parabola but with lower values because of less energy dissipation as explained before (Sec. 4.3). Since $\mu^{*}=1$, interfacial deformations alone are responsible for the onset of shear flows that create nonzero viscous torques on the cylinder and drive its rotation (see Sec. 4.1). The larger the interfacial deformations, i.e. when $\theta$ deviates from $90^{\circ}$, the faster the rotation, and the larger the drag reduction. In Fig. 7b, we see indeed that the ratio $F_{v, r}^{*} / F_{v, n r}^{*}$ reaches its minimum value when the cylinder angular velocity $\omega$ is maximum. But note that the effect is rather small here: $F_{v, r}^{*}$ and $F_{v, n r}^{*}$ differ by $3 \%$ at most. Hence, with $\mu^{*}=1$, interfacial deformations alone do not have a significant influence on particle rotation.

Moreover, Fig. 7b shows that the contact angle value controls the spinning direction of the cylinder: clockwise (resp., counter-clockwise) rotation with $\omega<0$ (resp., $\omega>0$ ) occurs for $\theta>90^{\circ}$ (resp., $\theta<90^{\circ}$ ), i.e. when the meniscus is deformed downwards (resp., upwards) and most of the particle body is exposed to the upper (resp., lower) fluid flow. The linear relationship between $\omega$ and $\theta$ simply arises from the fact that, in $2 \mathrm{D}$, the viscous torque $\left(\Gamma_{v, z}\right)$ exerted on the cylinder is, to a first approximation, a linear function of $\Delta L=L_{1}-L_{2}$, where $L_{1}$ (resp., $L_{2}$ ) is the contour length of the cylinder exposed to fluid 1 (resp., fluid 2). Since $L_{1}$ (or $L_{2}$ ) is proportional to the floating angle $\beta$ (Fig. 1b), and that $\beta=\theta-\zeta$, where $\zeta$ is the interface slope at the contact line (see Fig. 1b), it follows that $\Gamma_{v, z} \propto \theta$. Furthermore, since $R e \ll 1$ (Stokes regime) and $\mu^{*}=1, \Gamma_{v, z}$ is a linear function of $\omega$, as expected (see Fig. S4, SI), and therefore, $\omega \propto \theta$.

Finally, note that a linear relationship between the viscous torque and the vertical position of a particle across a flat fluid interface with $\mu^{*}=1$ was also reported in the 
3D calculations of Danov et al. [34]. In their study, the interface is undisturbed, but shifting the particle up or down across it also breaks the symmetry of the flow pattern and increases the dissipation, similarly to the curved menisci we have here, as pointed out in $[24]$.

\section{Concluding remarks}

In this work, we have probed, through $2 \mathrm{D}$ dynamic numerical simulations, the influence of particle rotation on capillary interactions. Two different problems and geometries were considered. In problem A, we focused on the capillary interaction between two particles attached to a fluid interface and able to both rotate and translate. The capillary attraction was driven by interfacial distortions caused by the particles' buoyant weight. The flow generated by the moving particles yields nonzero viscous torques which drive particle rotation. As a salient result of our investigation, we found that the speed of the capillary interaction can be significantly increased with rotating particles compared to non-rotating ones. A mismatch in the viscosity ratio between the two fluids enhances the kinetic boost observed in the rotating case. Our findings could be rationalized thanks to dedicated drag force computations (problem B) carried out with rotating and non-rotating particles trapped at a fluid interface. We found that the drag force exerted on a rotating particle was always less than its non-rotating counterpart because of attenuated velocity gradients in the vicinity of the particle. Furthermore, the larger the viscosity mismatch, the greater the difference due to an enhanced particle rotation. These results explain the faster interaction speed shown by rotating particles. However, for fluids with matched viscosities, the influence of interfacial deformations on particle rotation is minute.

Experimentally, capillary interactions occurring with both spinning and translating particles have not been reported so far, to the best our knowledge. A possible reason for this is that contact lines may be very easily pinned on nanoscopic defects of a solid surface due its roughness or chemical heterogeneities, as mentioned in the introduction $[16,17,32]$. Contact line pinning may prevent the particle from rotating, despite nonzero torques, if surface tension forces are sufficiently large. This is typically the case of liquid- 
gas interfaces, with which many capillary interaction experiments were carried out $[6,7]$. However, it would be interesting to design new experiments with 'smooth' enough particles to confirm our predictions. Perhaps particles coated with a thin fluid layer, i.e. with a kind of core-shell-like structure as in double emulsions designed via microfluidic tools [35], could serve as good candidates.

Finally, our work was limited to two dimensions, and therefore only applicable to particles with a simple shape (disc or cylinder per unit length). Extending the present study to the more general case of non-spherical particles would require full three-dimensional computations since these particles generally exhibit complex deformed contact lines when attached to fluid interfaces [6, 7]. More rotational degrees of freedom and nonzero capillary torques would have to be taken into account as well. We hope that the present work will foster the development of other numerical or theoretical studies capable of tackling similar issues at, for instance, complex fluids interfaces such as those based on liquid crystals $[36,37]$.

\section{Acknowledgements}

This work was financially supported by the EU Marie-Curie fellowship 'CoPEC' under grant No 794837-H2020-MSCA-IF-2017 and by the NSERC Discovery grant No 201904162. One of us (J.-C.L.) is also indebted to the University of Bordeaux for further financial support thanks to the IdEx program entitled "Développement des carrières Volet personnel de recherche". We acknowledge CMC Microsystems for software licensing. The IT staff of the Mathematics department of the University of British Columbia is also gratefully acknowledged for their valuable help and support.

\section{Author contribution statement}

J-CL and JJF designed the research. J-CL and JH performed the work; all authors analyzed the data, interpreted the results and collaborated to the manuscript written by J-CL. 


\section{References}

[1] Lotito V, Zambelli T (2017) Approaches to self-assembly of colloidal monolayers: A guide for nanotechnologists. Adv. Colloid Interf. Sci. 246:217-274.

[2] Kralchevsky PA, Nagayama K (2000) Capillary interactions between particles bound to interfaces, liquid films and biomembranes. Adv. Coll. Interf. Sci. 85:145-192.

[3] Oettel M, Dietrich S (2008) Colloidal Interactions at Fluid Interfaces. Langmuir 24(4):1425-1441.

[4] Danov KD, Kralchevsky PA (2010) Capillary forces between particles at a liquid interface: General theoretical approach and interactions between capillary multipoles. Adv. Coll. Interf. Sci. 154:91-103.

[5] Botto L, Lewandowski EP, Cavallaro M Jr, Stebe KJ (2012) Capillary interactions between anisotropic particles. Soft Matter 8:9957-9971.

[6] Dasgupta S, Aust T, Gompper G (2017) Nano- and microparticles at fluid and biological interfaces. J. Phys.: Condens. Matter 29(37):373003 (41pp).

[7] Liu IB, Sharifi-Mood N, Stebe KJ (2018) Capillary assembly of colloids: Interactions on planar and curved interfaces. Annu. Rev. Condens. Matter Phys. 9:283-305.

[8] McGorty R, Fung J, Kaz D, Manoharan VN (2010) Colloidal self-assembly at an interface. Materials Today 13(6):34-42.

[9] Xie Q, Davies GB, Harting J (2016) Controlled capillary assembly of magnetic Janus particles at fluid-fluid interfaces. Soft Matter 12:6566-6574.

[10] Vassileva ND, van den Ende D, Mugele F, Mellema J (2005) Capillary forces between spherical particles floating at a liquid-liquid interface. Langmuir 21:11190-11200.

[11] Boneva MP, Christov NC, Danov KD, Kralchevsky PA (2007) Effect of electric-field induced capillary attraction on the motion of particles at an oil-water interface. Phys. Chem. Chem. Phys. 9:6371-6384. 
[12] Boneva MP, Danov KD, Christov NC, Kralchevsky PA (2009) Attraction between particles at a liquid interface due to the interplay of gravity- and electric-field-induced interfacial deformations. Langmuir 25(16):9129-9139.

[13] Dani A, Keiser G, Yeganeh M, Maldarelli C (2015) Hydrodynamics of particles at an oil-water interface. Langmuir 31:13290-13302.

[14] Singh P, Joseph DD (2005) Fluid dynamics of floating particles. J. Fluid Mech. 530:31-80.

[15] Dörr A, Hardt S (2015) Driven particles at fluid interfaces acting as capillary dipoles. J. Fluid. Mech. 770:5-26.

[16] Kaz DM, McGorty R, Mani M, Brenner MP, Manoharan VN (2012) Physical ageing of the contact line on colloidal particles at liquid interfaces. Nature Materials 11:138142.

[17] Rahmani AM, Wang A, Manoharan VN, Colosqui CE (2016) Colloidal particle adsorption at liquid interfaces: capillary driven dynamics and thermally activated kinetics. Soft Matter 12:6365-6372.

[18] Das S, Koplik J, Farinato R, Nagaraj DR, Maldarelli C, Somasundaran P (2018) The translational and rotational dynamics of a colloid moving along the air-liquid interface of a thin film. Scientific Reports 8:8910-1-13.

[19] Seppecher P (1996) Moving contact lines in the Cahn-Hilliard theory. Int. J. Engng. Sci. 34(9):977-992.

[20] Jacqmin D (2000) Contact-line dynamics of a diffuse fluid interface. J. Fluid Mech. 402:57-88.

[21] Qian T, Wang XP, Sheng P (2006) Molecular hydrodynamics of the moving contact line in two-phase immiscible flows. Commun. Comput. Phys. 1:1-52.

[22] Yue P, Zhou C, Feng JJ (2010) Sharp-interface limit of the Cahn-Hilliard model for moving contact lines. J. Fluid Mech. 645:279-294. 
[23] Wörner M (2012) Numerical modeling of multiphase flows in microfluidics and micro process engineering: a review of methods and applications. Microfluid Nanofluid 12:841-886.

[24] Loudet JC, Qiu M, Hemauer J, Feng FF (2020) Drag force on a particle straddling a fluid interface: Influence of interfacial deformations. Eur. Phys. J. E 43:13, DOI 10.1140/epje/i2020-11936-1.

[25] Jacqmin D (1999) Calculation of two-phase Navier-Stokes flows using phase-field modeling. J. Comput. Phys. 155:96-127.

[26] Yue P, Feng JJ, Liu C, Shen J (2004) A diffuse-interface method for simulating two-phase flows of complex fluids. J. Fluid Mech. 515:293-317.

[27] Pigeonneau F, Hachem E, Saramito P (2019) Discontinuous Galerkin finite element method applied to the coupled unsteady Stokes/Cahn-Hilliard equations. Int. J. Numer. Meth. Fluids 90:267-295.

[28] Yue P, Zhou C, Feng JJ, Ollivier-Gooch CF, Hu HH (2006) Phase-field simulations of interfacial dynamics in viscoelastic fluids using finite elements with adaptive meshing. J. Comput. Phys. 219:47-67.

[29] Singh P, Hesla TI (2004) The interfacial torque on a partially submerged sphere. J. Colloid Interface Sci. 280:542-543.

[30] COMSOL Multiphysics ${ }^{\circledR}$ v. 5.4 reference manual. See https://www.comsol.com/ documentation. COMSOL AB, Stockholm, Sweden.

[31] Khalili A, Liu B (2017) Stokes' paradox: creeping flow past a two-dimensional cylinder in an infinite domain. J. Fluid Mech. 817:374-387.

[32] Koplik J, Maldarelli C (2017) Diffusivity and hydrodynamic drag of nanoparticles at a vapor-liquid interface. Phys. Rev. Fluids 2:024303-1-12.

[33] Deen WM, Analysis of transport phenomena, Oxford University Press: Oxford, UK, 1998. 
[34] Danov KD, Dimova R, Pouligny B (2000) Viscous drag of a solid sphere straddling a spherical or flat interface. Physics of Fluids 12:2711-2722.

[35] Nawar S, Stolaroff JK, Ye C, Wu H, Nguyen DT, Xin F, Weitz DA (2020) Parallelizable microfluidic dropmakers with multilayer geometry for the generation of double emulsions. Lab on a Chip 20:147-154.

[36] Oettel M, Domínguez A, Tasinchevych M, Dietrich S (2009) Effective interactions of colloids on nematic films. Eur. Phys. J. E 28:99-111.

[37] Liu IB, Gharbi MA, Ngo VL, Kamien RD, Yang S, Stebe KJ (2015) Elastocapillary interactions on nematic films. Proc. Natl. Acad. Sci. USA 112:6336-6340. 